\title{
Whole-brain microcirculation detection after ischemic stroke based on swept-source optical coherence tomography
}

\author{
Jian Liu ${ }^{1}$ | Ning Ding ${ }^{2}$ | Yao Yu ${ }^{1}$ | Lanxiang Liu ${ }^{3}$ | Xincheng Yuan ${ }^{4}$ | Hongyu Lv ${ }^{5}$ | \\ Yuqian Zhao ${ }^{1}$ I Zhenhe Ma ${ }^{1 *}$ ()
}

\author{
${ }^{1}$ School of Control Engineering, \\ Northeastern University at Qinhuangdao, \\ Qinhuangdao, China \\ ${ }^{2}$ School of Sino-Dutch Biomedical and \\ Information Engineering, Northeastern \\ University, Shenyang, China \\ ${ }^{3}$ Department of Magnetic Resonance \\ Imaging, Qinhuangdao Municipal \\ No. 1 Hospital, Qinhuangdao, China \\ ${ }^{4}$ Department of Biomedical Engineering, \\ University of Michigan, Ann Arbor, \\ Michigan \\ ${ }^{5}$ Department of Ophthalmology, Maternal \\ and Child Health Hospital, Qinhuangdao, \\ China
}

\section{*Correspondence}

Zhenhe Ma, School of Control Engineering, Northeastern University at Qinhuangdao, Qinhuangdao 066004, China.

Email: mazhenhe@163.com

\section{Funding information}

Fundamental Research Funds for the Central Universities, Grant/Award Numbers: N182304008, N172304034, N172304029, 17277718D; Technology Supporting Program of Hebei Province, Grant/Award Number: 17277718D; Hebei Provincial Natural Science Foundation of China, Grant/ Award Number: H2018501087; National Natural Science Foundation of China, Grant/ Award Numbers: 61771119, 81871029

\begin{abstract}
The occurrence and development of ischemic stroke are closely related to cerebral blood flow. Real-time monitoring of cerebral perfusion level is very useful for understanding the mechanisms of the disease. A wide field of view (FOV) is conducive to capturing lesions and observing the progression of the disease. In this paper, we attempt to monitor the
\end{abstract}

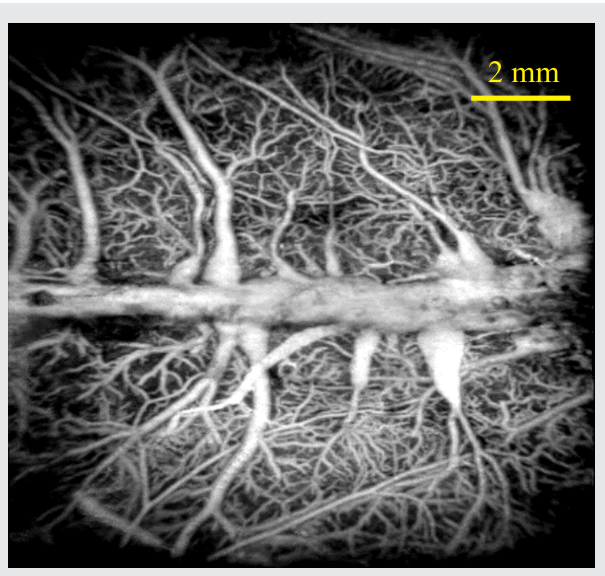
whole-brain microcirculation in middle cerebral artery occlusion (MCAO) rats over time using a wide FOV swept-source OCT (SS-OCT) system. A constrained image registration algorithm is used to remove motion artifacts that are prone to occur in a wide FOV angiography. During ischemia, cerebral perfusion levels in the left and right hemispheres, as well as in the whole brain were quantified and compared. Changes in the shape and location of blood vessels were also recorded. The results showed that the trend in cerebral perfusion levels of both hemispheres was highly consistent during MCAO, and the position of the blood vessels varied over time. This work will provide new insights of ischemic stroke and is helpful to assess the effectiveness of potential treatment strategies. (C) 2019 WILEY-VCH Verlag GmbH \& Co. KGaA, Weinheim

\section{K E Y W O R D S}

constrained image registration, ischemic stroke, swept-source optical coherence tomography, wide field of view

\section{1 | INTRODUCTION}

Ischemic stroke, a severe acute neurological disorder, can give rise to irreversible damage to brain tissue and have a tremendous impact on the subsequent quality of life for the victims $[1,2]$. This disease occurs when the blood that flows into parts of the brain is interrupted or reduced, depriving brain tissue of oxygen and nutrients, followed by brain cells beginning to die within minutes [3]. Obviously, the occurrence and development of ischemic stroke are closely related to cerebral blood flow. Real-time monitoring of cerebral blood flow perfusion 
level is very useful for understanding the mechanisms of the disease.

Nowadays, rodent models are indispensable in deepening our understanding of human disease. The use of appropriate animal models is essential for evaluating various therapies for human subjects. For cerebrovascular imaging of rodents in vivo, a non-invasive high-speed 3D imaging tool with micron-level resolution is needed [4].

Optical coherence tomography (OCT) is a non-invasive method that allows volumetric imaging of biological tissues at high resolution, great depth and high speed [5]. OCTbased angiography (OCTA) is a method of visualizing cerebral perfusion levels by distinguishing moving particles from static tissues without the need for contrast agents, which extend the application of OCT from purely structured imaging to functional imaging [6]. Middle cerebral artery occlusion (MCAO) often leads to extensive ischemia in the cortex, and its damage may vary greatly depending on individual differences. Narrow FOV imaging is not conducive to capturing lesions and accurately mastering conditions. In addition, most cerebral ischemia occurs in the unilateral hemisphere. In clinical or basic research, the comparison between the left and right hemispheres is often the most intuitive method to determine the degree of ischemia. Therefore, a whole-brain FOV, high-quality OCT imaging is the goal pursued by scholars. Traditional spectral-domain optical coherence tomography (SD-OCT), although it is capable of providing wide FOV imaging [7], suffers from low spectral resolution, resulting in a rapid fall-off in sensitivity along depth [8]. Rodent brains have a natural curvature, and there is a significant depth difference $(>5 \mathrm{~mm}$ ) between the anterior cerebral artery (ACA) side and the middle cerebral artery (MCA) side. Using SD-OCT to image the entire cerebral vascular network of small animals may cause excessively low sensitivity in the deeper region on both sides of the brain which thus makes the image blurry. One feasible approach is to make multiple acquisitions and then merge the generated images, which nonetheless is time-consuming and cumbersome [9]. Moreover, the images produced by each acquisition may be inconsistent due to the readjustment of the sample.

Recent developed swept-source optical coherence tomography (SS-OCT) has several significant advantages over SDOCT, including greater light intensity and higher spectral resolution. Hence, SS-OCT has a deeper penetration depth and a smaller sensitivity fall-off along the depth. These advantages enable SS-OCT to better cope with rugged samples in wide FOV imaging. Numerous research groups reported on SS-OCT and its applications. Song et al designed a SS-OCT system with an unprecedented FOV (up to $400 \mathrm{~cm}^{2}$ ) for in vivo imaging of human hands and faces [10]. Xu et al developed a wide-field and highly sensitive SS-OCT system that enables extra-long imaging depth (up to $46 \mathrm{~mm}$ ) in microcirculatory tissue beds. Additionally, they demonstrated unprecedented quality blood perfusion maps for human fingers through a single 3D scan with the system [11]. Zhongwei Zhi et al demonstrated the capability of an ultrahigh-speed (1.6 MHz) SS-OCT system to produce $4 \mathrm{D}$ in vivo optical microangiography of tissue with a volume rate of 4.7 volumes/s [12]. SS-OCT is also widely used in clinical settings, such as ophthalmology [13-16], dermatology [17], oral and nasal cavities [18]. OCT angiography is based on motion contrast, and the risk of motion artifacts increases with the increase of imaging range. Motion artifacts can greatly affect the quality of angiograms and the quantification of blood perfusion, so they must be eliminated. At present, some motion artifact elimination techniques have been developed. For example, triggering the acquisition based on the heart rate [19], but this method requires additional hardware additions; or acquiring a large number of B-scans at the same location and averaging them together [20, 21], however, this method increases the total acquisition time. Also, it is possible to remove the frames with motion artifacts that exceed a certain threshold [22], which wastes data acquisition time at the expense of image signal-to-noise ratio (SNR). Image registration methods have also been used [23], but the conventional image registration algorithm is likely to cause "over registration" of the position where no motion occurs, dimming of blood vessel images, and affecting the quantification of blood perfusion. So far, research to eliminate motion artifacts continues [24, 25].

In this paper, we attempt to monitor the whole brain microcirculation in MCAO rats over time using wide FOV SS-OCT system. A constrained image registration algorithm is used to remove motion artifacts. Cerebral perfusion levels in the left and right hemispheres and the whole brain were quantified and compared during the setting of ischemia. The changes in the shape and location of the blood vessels were also recorded. This work will provide new insights of the pathology of ischemic stroke and is helpful to evaluation of effectiveness of potential treatment strategies.

\section{2 | METHOD}

\section{1 | System}

In this work, we set up a wide-range SS-OCT system, as shown in Figure 1. The light source employed is an akinetic swept-source (MEMS-VCSEL, Thorlabs Inc, USA), operating at $200 \mathrm{kHz}$ swept rate, with a central wavelength of $1300 \mathrm{~nm}$, a bandwidth of $100 \mathrm{~nm}$, and an axial resolution of approximately $7.5 \mu \mathrm{m}$ in air. The beam emitted by the swept source was split into the sample arm and the reference arm by a 90:10 ratio coupler (TW1300R2A2, Thorlabs Inc). In 


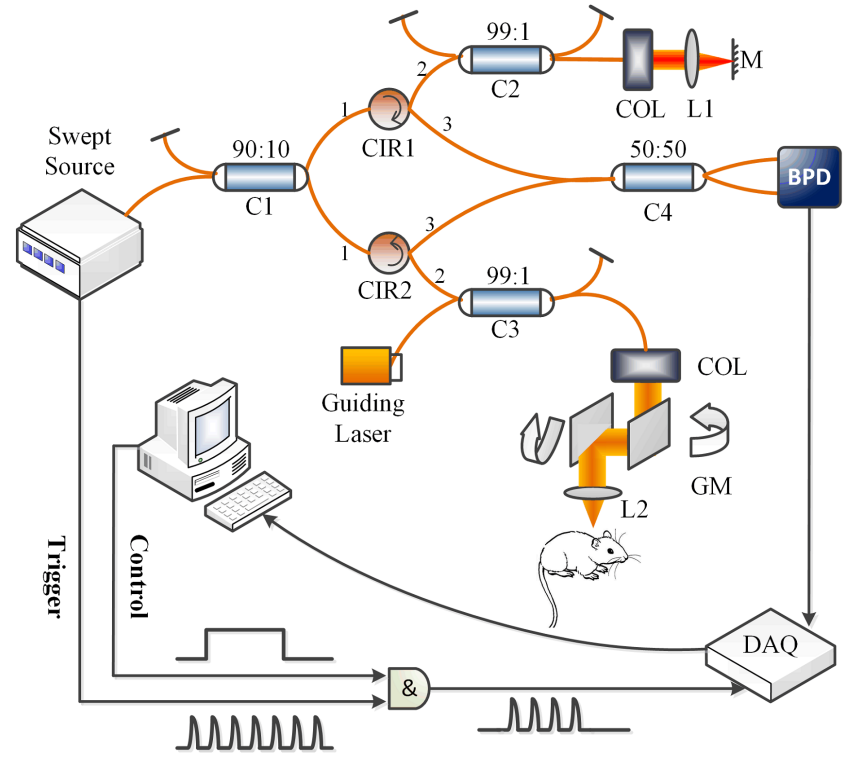

F I G U R E 1 Wide-range swept-source OCT system. L1, L2: Lens; C1, C2, C3, C4: fiber coupler; BPD: balanced photo detector; M: mirror; COL: collimator; CIR1, CIR2: circulator; GM: galvanometric mirror

the sample arm, an aiming beam was combined with another 99:1 coupler to guide the OCT imaging. A 50-mm focal length objective lens (AC254-050-C, Thorlabs Inc) was used in the sample arm to achieve the lateral resolution of approximately $16 \mu \mathrm{m}$. A pair of XY galvanometric mirrors were used in the system to form a 3D scan. In order to control both the galvanometric mirror and the acquisition synchronization, we added an AND gate circuit to the system. An AND operation was performed to the triggering signal from the swept source and the control signal (square wave) and the resultant signal was used to control data acquisition. A high-speed dual-balanced photodetector (Thorlabs, PDB480C-AC) with $1.6 \mathrm{GHz}$ bandwidth and a super data sampling digital card (PXLe-5162, National Instruments, USA) with $5 \mathrm{GHz}$ sampling rate were used to detect the OCT interferograms, which provided a spectral resolution $R_{\lambda}$ of $0.004 \mathrm{~nm}$. The theoretical ranging depth is given by $Z_{\max }=\lambda^{2} /\left(4 n R_{\lambda}\right)$ [8], where $n$ is the refractive index of the sample and $\lambda$ is the central wavelength. The theoretical ranging depth of the system can reach approximately $78 \mathrm{~mm}$. Imaging of rat cerebral cortex does not require such depth of detection, so each interference spectrum was digitized to 5000 points, which provided an actual measurement depth of approximately $16 \mathrm{~mm}$. Finally, an adaptive calibration procedure proposed by Shaozhen Song et al was used in the correction of the SS-OCT spectral [10].

\section{2 | OCTA protocol}

OCTA utilizes the intrinsic motion contrast caused by dynamically moving particles (eg, red blood cells) to differentiate functional blood vessels from static tissue background. In our system, the fast scanner (X direction) was driven by a saw tooth waveform with a $160 \mathrm{~Hz}$ frame rate, providing $6.25 \mathrm{~ms}$ time interval between adjacent repeated B-scans. Each B-scan image contained 1000 A-lines that occupied $80 \%$ duty cycle. The slow scanner (Y direction) was driven by a step function waveform, which led to 800 B-scan positions with four repeated B-scans per step. The FOV is $12 \mathrm{~mm}$ ( $\mathrm{X}$ direction) $\times 10 \mathrm{~mm}$ (Y direction), enough to cover the entire cerebral cortex of the rats. Therefore, each data set of the OMAG contained 3200000 Alines, taking 20 seconds to complete the data acquisition.

Most current swept sources suffer from severe trigger jitter and phase instability due to the variation of mechanical movement, which reduces system reproducibility. In particular, intensity-signal-based OCTA does not require a phasestabilized system and thus can be used with phase-instable systems such as SS-OCT. By comparing the performance of intensity-signal-based OCTA with traditional phase-resolved Doppler variance and that of color Doppler methods, Liu et al concluded that the former demonstrated good performance in both phase-stable and phase-instable cases [26]. In this paper, a differential operation was performed for intensity signals acquired at the same location at different time points to distinguish moving particles from static tissue. In the process of data acquisition, if small animals move, motion artifacts will occur.

\section{3 | Motion artifact elimination}

Performing image registration for repeated B-scans at the same location is an effective way to eliminate motion artifacts. According to our previous research, an efficient nonparametric diffeomorphic image registration algorithm based on Thirion's demons proposed by Tom Vercauteren et al has been proven to be suitable for non-rigid transformation of soft tissue images, such as small animal blood vessels [27, 28]. The algorithm can effectively remove motion artifacts, realize the clarification of vascular images, and contribute to the further analysis of cerebrovascular diseases in small animals. Figure 2A shows an angiogram with motion artifacts (blue arrows). In order to increase the intuitiveness of the image, we added pseudo color. Figure 2B is an angiogram after registration based on Thirion's demons. However, there are still shortcomings in this approach. Some locations where there is no movement produced an "over registration" (green arrow), darkening the angiogram and affecting the quantification of blood perfusion. To solve this problem, we propose an image registration algorithm based on constrained demons. We repeatedly collected four B-scans at each position, using the first B-scan as the reference image, and registering the next three B-scans with the first B-scan. A similarity test is 
(a)

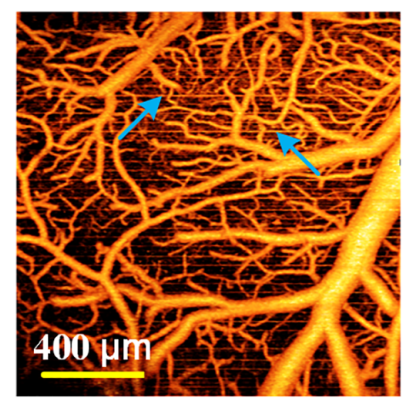

(b)

(c)

(d)
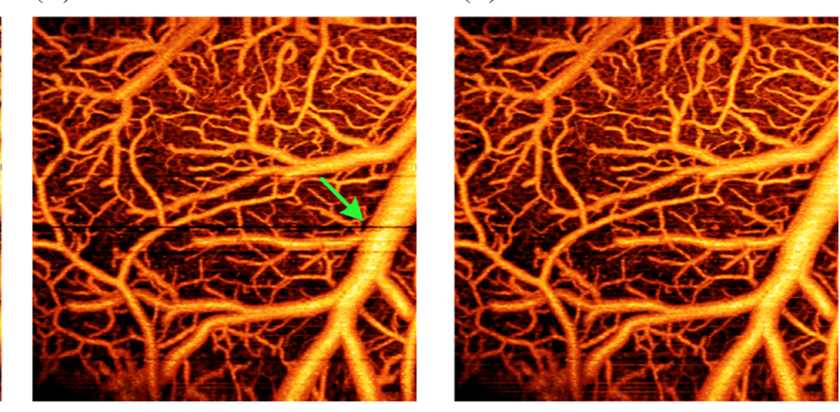

F I GURE 2 Image registration method. A, An angiogram with motion artifacts (blue arrow). B, The angiogram after registration based on Thirion's demons, the green arrow shows the "over registration" phenomenon. $\mathrm{C}$, The angiogram after registration based on constrained demons. D, Correlation coefficients between the motion and no motion adjacent $\mathrm{B}$-scans, the best threshold is 0.92

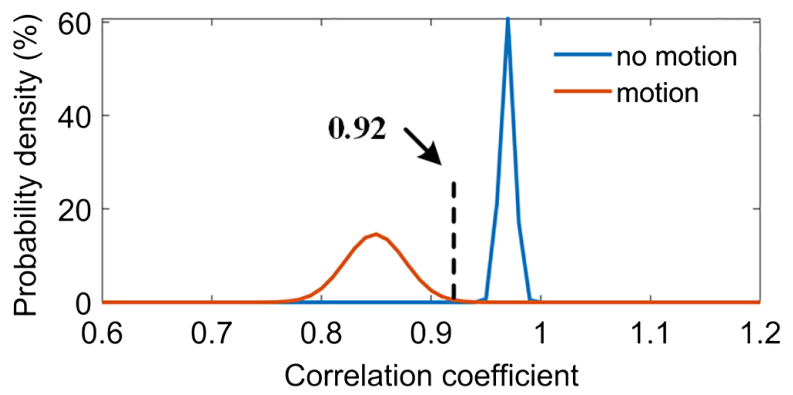

performed prior to registration and then determines if they need to be registered according to the test results.

The method is to calculate the correlation coefficient between the reference image and the image to be registered, and then a suitable threshold is used. Frames only whose correlation coefficient is smaller than the threshold are selected to perform registration. Now, the threshold becomes the key to the problem.

We separately calculated the correlation coefficient with and without motion $(\mathrm{n}=100)$. Two probability density function (PDF) curves were drawn, as shown in Figure 2D. The blue curve indicates that no motion has occurred, while the red represents the case of motion. From them, we found that the correlation coefficient for no motion was about $97 \%$, and that for motion was about $85 \%$; According to the minimum error thresholding (MET) criteria [29], we set the threshold to $92 \%$.

The registration result is shown in Figure 2C. The proposed method can effectively remove artifacts, limit the occurrence of "over registration" phenomenon, and at the same time reduce the amount of calculation, and improve the operation speed.

\subsection{Quantification of cerebral perfusion level}

The cerebral vessel perfusion density (CVPD), defined as the ratio of "pixels of blood vessels with flow" to "pixels of the entire blood vessel image", is an indicator of cerebral perfusion level. In this paper, CVPD was calculated using "locally adaptive region growing algorithm" proposed previously [30]. Briefly, the algorithm consists of four steps: (a) The selection of initial seed pixels; (b) Local region selection; (c) Threshold update; (d) Similarity judgment. Firstly, initial seed pixels were automatically selected with a certain gray value in an image histogram. Then, a local region around the initial seed pixel was determined based on vessel size, and a threshold was calculated based on the value of seed pixels. Within the neighborhood of the initial seed pixel, to which candidate pixels having similarity will be incorporated into the object region (here, the object region represents a blood vessel). The similarity here indicates that the difference between two pixels is less than the threshold updated constantly. Based on this growth condition, the algorithm was repeated and terminated when there were no absorbable pixels. This algorithm is characterized by automatic adjustment of local growth criterion in different regions, as well as the ability to extract pixels from almost all blood vessels from unevenly illuminated OCT angiograms.

\section{5 | Animal model}

In this study, Three-month-old Sprague-Dawley rats (male) were used, each weighing approximately $250 \mathrm{~g}$. All procedures were performed in accordance with the Animal Ethics and Administrative Council of Northeastern University. All efforts were made to minimize animal suffering and to reduce the number of animals used. Surgical anesthesia was induced with sodium pentobarbital $(3 \%, 5 \mathrm{mg} / 100 \mathrm{~g}$, IP). The anesthetized rats were fixed on a stereotaxic apparatus (ST-5ND-C) with ear bars and a clamping device. The fur on the rats' heads was shaved, and the skin was cleaned with saline. The skin was cut along the midline of the skull, and the interparietal bone was exposed by pulling the skin to both sides. The size of the exposed interparietal bone is 
approximately $10 \mathrm{~mm}$ (anterior and posterior direction) $\times$ $8 \mathrm{~mm}$ (lateral direction). The FOV provided by our system $(12 \mathrm{~mm} \times 10 \mathrm{~mm})$ can cover the entire interparietal bone. Next, subcutaneous tissue and the periosteum were cleaned. To obtain a clearer image, we ground the skull to a suitable thickness using a $1.2 \mathrm{~mm}$ diameter flat cranial drill. After the procedure, the rat was placed in the arm of the OCT sample and prepared for data acquisition (baseline).

The model of cerebral ischemia used in our experiments was based on endothelin-1-induced middle cerebral artery occlusion (MCAO) model described previously [31, 32]. The stereotaxic coordinates of the ET-1 injection were $0.9 \mathrm{~mm}$ anterior, $5.2 \mathrm{~mm}$ lateral and $8.7 \mathrm{~mm}$ ventral relative to bregma. A dose of $3 \mu \mathrm{L} \mathrm{ET-1} \mathrm{(60} \mathrm{pmol/L} \mathrm{diluted} \mathrm{with}$ saline) was injected at $1 \mu \mathrm{L} / \mathrm{min}$. After injection, we waited for 3 minutes to ensure that the drug was fully absorbed. The needle was slowly removed, and the rat was immediately placed under the sample arm for scanning.

\section{3 | RESULT}

The entire cerebrovascular network was collected with only one scan by the SS-OCT system. Figure 3A shows en face maximum intensity projection (MIP) of the vascular network in cortical layers of a rat brain (up to $300 \mu \mathrm{m}$ depth) at baseline (ie, before ET-1 injection), while Figure 3B-D shows the MIPs at 15, 45 and 120 min after ET-1 injection, respectively. As shown in Figure 3A, the system provided clear visualization of the vascular network and associated networks in the middle cerebral arteries (MCA) domain, which are located in relatively low positions on the left and right sides of the rat brain. After ET-1 injection, the blood flow of large blood vessels and capillaries in the MCA area decreased dramatically, as indicated by the observed lack of blood vessels. Figure 3E-H shows the corresponding vascular segmentation results obtained by our previously proposed method (locally adaptive region growing algorithm).

CVPD was calculated based on the segmentation results. Each $1000 \times 800$ pixel image was divided into two $1000 \times 400$ pixel images from the middle longitudinal line, resulting in the ipsilateral hemisphere (ischemic side) and the contralateral hemisphere (healthy side), respectively. Four MCAO rats were used in the experiment, and statistics was performed on the ipsilateral hemisphere, contralateral hemisphere and the whole brain. Three time-varying CVPD curves were obtained, as shown in Figure 4.

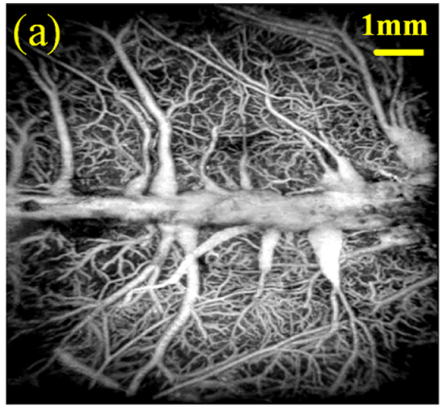

$15 \min$
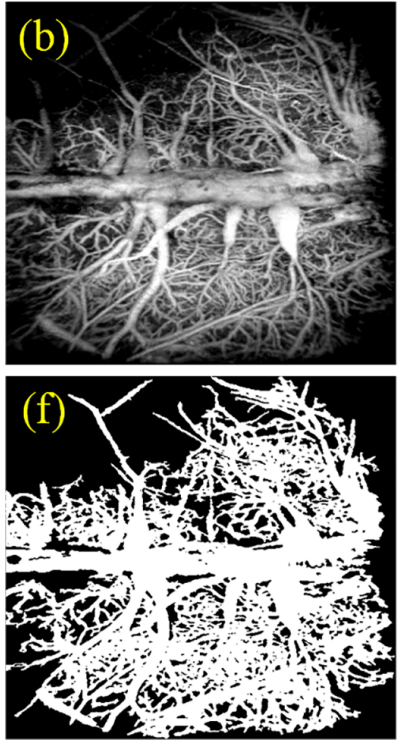

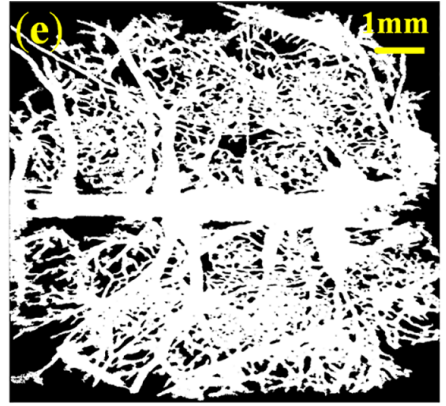

Baseline

$45 \mathrm{~min}$
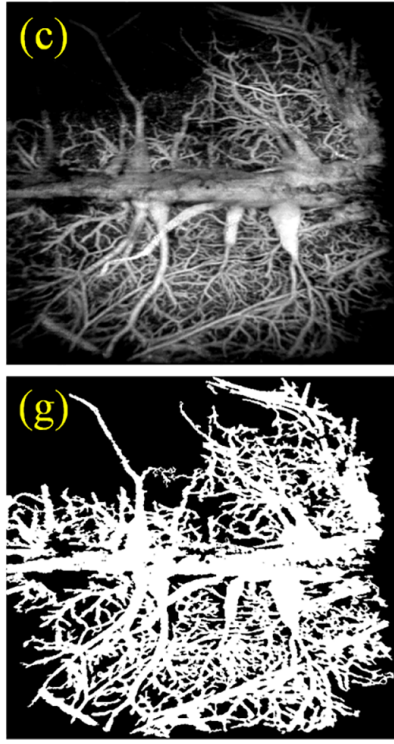
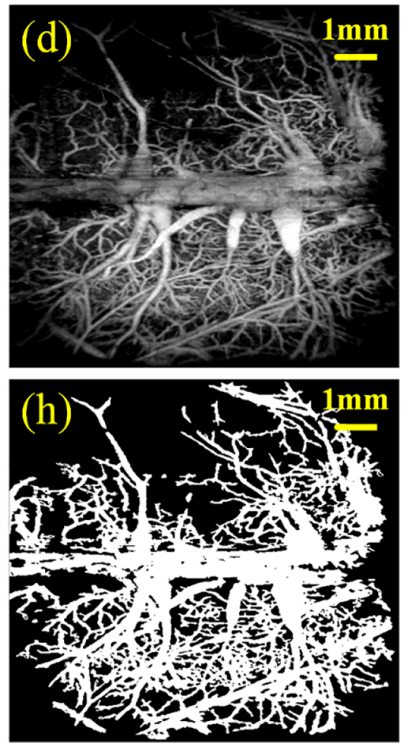

FIGURE 3 En face maximum intensity projections (MIP) of the vascular networks (A-D) and the corresponding blood vessel segmentation results $(\mathrm{E}-\mathrm{H})$, before and after MCAO 


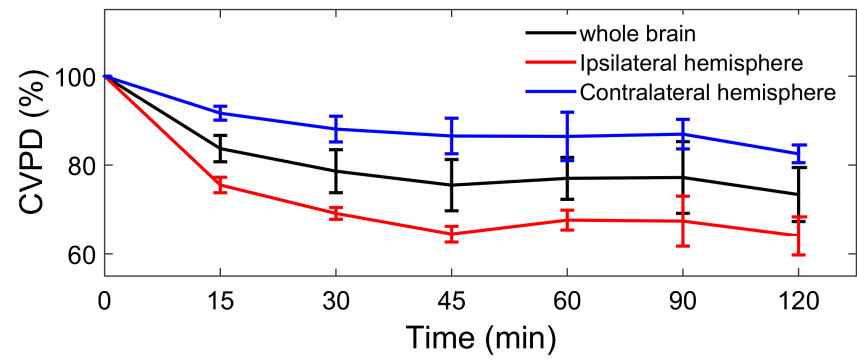

F I G URE 4 Percentage change in CVPD in the ipsilateral hemisphere, the contralateral hemisphere and the whole brain, respectively. Error bars represent standard error $(n=4)$

As shown in Figure 4, all three CVPD curves decreased over time. Specifically, the curve representing ipsilateral hemisphere declined the fastest in the initial 15 minutes, followed by a marked slowdown. At 45 minutes, the percentage dropped to only $64 \%$ of the baseline. It then rose slightly until 90 minutes and they started to decrease again. It can also be seen that the CVPD curve of the contralateral hemisphere showed a similar trend, falling to $82.5 \%$ of the baseline within 120 minutes. The results of the four rats were consistent with each other.

In order to explain this phenomenon, we performed quantitative analysis of contralateral hemisphere vessel diameter. We randomly selected seven contralateral hemisphere vessels (mainly large vessels) and measured the diameter of these vessels at different time points, expressed percentages. The result is shown in Figure 5.

It can be seen that the change of vascular diameter in the contralateral hemisphere is consistent with in the CVPD changes in the corresponding hemisphere. Within the first 45 minutes, vessel diameter experienced a continuous decrease, after which it increased until 90 minutes. The other three rats showed similar changes.

During the ischemic process, we found that the positions of the blood vessels changed over time. We suspect this is an occupancy effect caused by cerebral edema (a complications of stroke). The occupying effect of cerebral edema would compress the surrounding blood vessels, causing the blood vessels to shift and the diameter of the blood

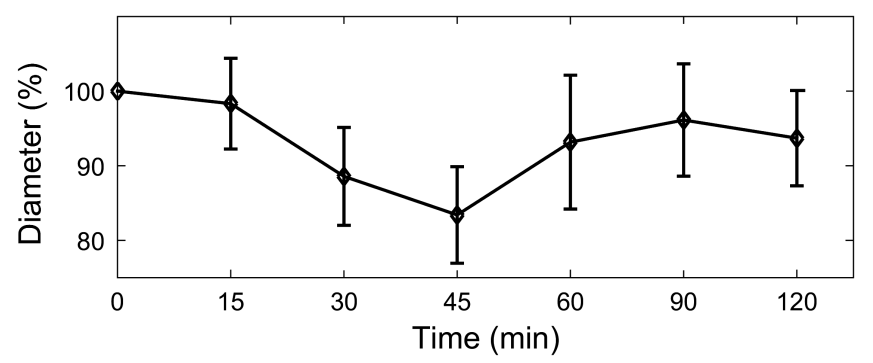

F I G URE 5 Diameter of the blood vessels in the contralateral hemisphere. Error bars represent standard error $(n=7)$ vessels to decrease, resulting in further ischemia of the cortex [33]. Assessing the occupancy effect of edema is of great significance in evaluating the degree of cerebral edema progression. In this paper, a simple path tracking method was used to study the occupancy effect of cerebral edema. A certain number of feature points were selected from an OCT angiography. The locations of each point at different times are recorded, respectively, to generate the trajectory of the feature point. Obviously, such trajectory represents the displacement of compressed vessels. In this study, each feature point was manually selected from the intersection of branches of a vessel.

The result is demonstrated in Figure 6A shows three OCT angiograms for the same location at 30, 60 and 120 minutes, respectively. The red rectangle represents a region where vascular displacement is observed, while the yellow arrows indicate vessels of that type. A number of feature points were selected manually from vascular branch point in three different areas, named center, vicinity and periphery, denoted in red, yellow and blue, respectively. The position of each feature point at different times was recorded and shown in the last angiogram (120 minutes), as depicted in Figure 6B. For each point, its time-varying positions were
$30 \mathrm{~min}$

(a)

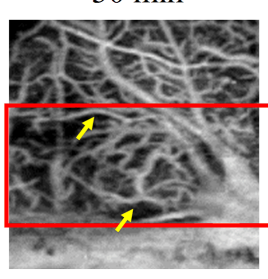

$60 \mathrm{~min}$

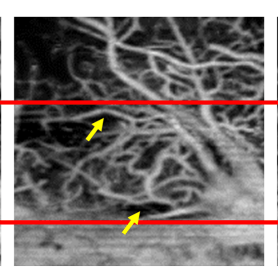

$120 \mathrm{~min}$

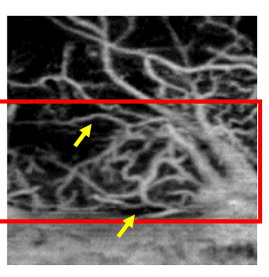

(b)

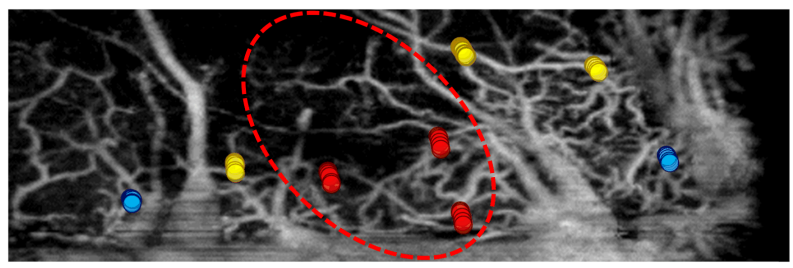

(c)

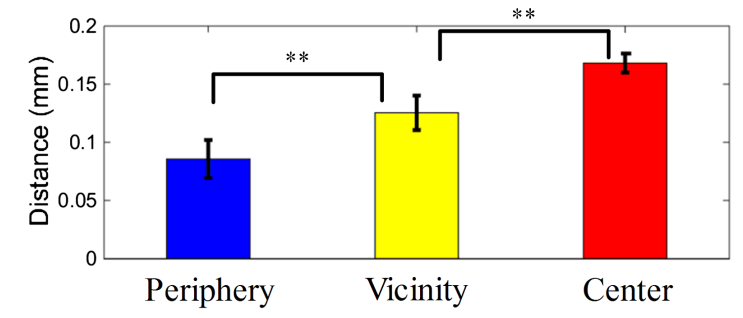

F I G URE 6 Schematic diagram of vascular displacement. A, Three OCT angiograms at 30,60 and 120 minutes, respectively. The red rectangle represents a region where vascular displacement is observable while the yellow arrows indicate vessels of that type. B, Path of blood vessel movement. Red ellipse indicates ischemia center, and Red, green and yellow points are blood vessel branches in three different areas, namely center, vicinity and periphery. The distance they moved is shown in $(\mathrm{C})$. ** represents $P<.01$ 
used to generate a trajectory of it over time as a result of vessel extrusion. Note that the center feature points (red) has the largest displacement $(0.17 \pm 0.02 \mathrm{~mm})$, with the displacement of the vicinity ones (yellow) second $(0.13 \pm 0.03 \mathrm{~mm})$ and the periphery ones (blue) with the smallest displacement $(0.09 \pm 0.04 \mathrm{~mm})$, as demonstrated in Figure 6C. We estimated the difference between different regions using $t$ test. Significant differences in distance of vascular displacement between different regions were found $(P<.01)$. Due to individual differences, there are certain variations between the vascular displacements of different rats, but their overall trends are similar.

\section{4 | DISCUSSION}

In this article, we report some preliminary results of wholebrain blood flow status after ischemic stroke. Through employing wide-FOV SS-OCT, changes in blood flow perfusion level and in vascular displacement throughout the brain were able to be observed. From the experimental results, we found some phenomena. One is that the trends of perfusion levels in both hemispheres are highly consistent during MCAO, suggesting that ischemia in one hemisphere would affect the state of blood flow in the other hemisphere. An earlier study found that in the contralateral hemisphere after a stroke, the CBF decreased in patients with unilateral hemispheric infarction and unilateral cerebrovascular disease $(P<.05)$. Multiple regression analysis demonstrated a high correlation between contralateral flow reduction and the degree of flow impairment in the infarcted area $(r=.941$, $P=.0014)$ but not with age, risk factor profile, blood pressure, $P_{\mathrm{CO} 2}$, hematocrit, or duration of stroke [34]. Another study showed that the CBF in the contralateral hemisphere decreased significantly by $35 \%$, compared to the baseline, 8 hours after ischemia, and this CBF reduction appeared to involve the entire brain. They hypothesized that diaschisis could be the basic pathophysiological process that can explain this global decrease in CBF [35]. Some scholars believe that the reduction of blood flow to the contralateral hemisphere is related to collateral circulation. Nevertheless, the mechanism behind it has not yet been understood.

From the results of this paper, we also found the phenomenon of vascular displacement. We suspect that this is caused by cerebral edema. Brain edema is one of the major complications of ischemic stroke, and it causes most deaths within the first week of the disease. Cerebral edema caused by cerebral ischemia can be categorized into either cytotoxic or vasogenic type [36]. The earliest phase of cerebral edema is characterized by cytotoxic edema, where ions move between extra- and intra-cellular spaces, and later along the resultant osmotic gradient [37]. This phase occurs in the first few hours of ischemia when the blood-brain barrier remains intact. The second phase, characterized by vasogenic edema, however, occurs after the blood-brain barrier is disrupted. During this phase, the permeability of macromolecules increases significantly, and consequently both hydrostatic and penetrating forces play increasingly important roles [38]. In both hemispheres, there is a correlation between vasogenic edema and increased water content and vascular volume. When cerebral edema develops, due to limited intracranial space, local swelling of brain tissue squeezes the surrounding tissues, resulting in ischemia. If medical intervention is not taken in a timely manner, there will be a vicious circle of cerebral edema, ischemia and cerebral edema, which in turn can lead to life-threatening cerebral hernia [39].

The aforementioned oscillation in CVPD and in vessel diameter could be a sign of the vicious circle for brain tissue. In order to explore the interaction between cerebral edema on cerebral vessels, we further studied space occupying effect of the former. In most previous studies, the quantification of such effect was related to the measurement of brain volume (such as MRI). In contrast, the quantification method proposed in this paper is based on vascular morphology. In Figure 6, it can be concluded that the region surrounded by the red dashed line identifies the area where space occupying effect is most severe in terms of vascular displacement of the feature points; also in addition, ischemia is the most severe in this region, so there is likely to be a strong correlation between cerebral edema and vascular displacement. The capillary diameter of mouse cerebral cortex is $5-8 \mu \mathrm{m}$, while the largest vascular displacement was approximately $170 \mu \mathrm{m}$. Therefore, this displacement is likely to lead to a huge impact on cerebral microcirculation. At the same time, this effect may also be related to a reduction in blood supply in the other hemisphere.

In summary, with whole-brain FOV and capillary resolution, the SS-OCT technique is a reliable tool for studying cerebrovascular diseases and evaluating potential treatment strategies.

\section{ACKNOWLEDGMENTS}

This work was supported in part by National Natural Science Foundation of China (61771119 and 81871029), Hebei Provincial Natural Science Foundation of China (H201850 1087). Fundamental Research Funds for the Central Universities (N182304008, N172304034 and N172304029), Technology Supporting Program of Hebei Province (1727 7718D).

\section{CONFLICT OF INTEREST}

The authors declare no potential conflict of interest. 


\section{AUTHOR BIOGRAPHIES}

Please see Supporting Information online.

\section{ORCID}

\section{Zhenhe Ma (1) https://orcid.org/0000-0003-4079-9550}

\section{REFERENCES}

[1] L. Yu, E. Nguyen, G. Liu, B. Choi, Z. Chen, J. Biomed. Opt. 2010, 15(6), 066006

[2] L. Mogoantă, D. Pirici, O. T. Pop, A. T. Bălşeanu, E. Rolea, R. M. Dahnovici, Romanian J. Morphol. Embryol. 2010, 51 (4), 725.

[3] O. D. Nurhayati, I. P. Windasari, Int. Conf. Inform. Technol. IEEE. 2016.

[4] U. Baran, R. K. Wang, Neurophotonics 2016, 3(1), 010902.

[5] R. K. Wang, IEEE J. Select. Topics Quantum Electron. 2010, 16 (3), 545 .

[6] R. K. Wang, L. An, S. Saunders, D. J. Wilson, J. Biomed. Opt. 2010, 15(2), 020502.

[7] J. Xu, W. Wei, S. Song, X. Qi, R. K. Wang, Biomed. Opt. Express 2016, 7(5), 1905.

[8] P. H. Tomlins, R. K. Wang, J. Phys. D. Appl. Phys. 2005, 38(15), 2519.

[9] Q. Q. Zhang, C. S. Lee, J. Chao, C. L. Chen, T. Zhang, U. Sharma, A. Q. Zhang, J. Liu, K. Rezaei, K. L. Pepple, R. Munsen, J. Kinyoun, M. Johnstone, R. N. Van Gelder, R. K. K. Wang, Sci. Rep. 2016, 6, 22017.

[10] S. Song, J. Xu, R. K. Wang, Biomed. Opt. Express 2016, 7(11), 4734.

[11] J. Xu, S. Song, W. Wei, R. K. Wang, Biomed. Opt. Express 2017 , $8(1), 420$.

[12] Z. Zhi, W. Qin, J. Wang, W. Wei, R. K. Wang, Opt. Lett. 2015, 40(8), 1779.

[13] I. Grulkowski, J. J. Liu, B. Potsaid, V. Jayaraman, C. D. Lu, J. Jiang, J. G. Fujimoto, Biomed. Opt. Express 2012, 3(11), 2733.

[14] I. Grulkowski, J. J. Liu, B. Potsaid, V. Jayaraman, J. Jiang, J. G. Fujimoto, A. E. Cable, Opt. Lett. 2013, 38(5), 673.

[15] J. J. Liu, I. Grulkowski, M. F. Kraus, B. Potsaid, C. D. Lu, B. Baumann, J. S. Duker, J. Hornegger, J. G. Fujimoto, Biomed. Opt. Express 2013, 4(2), 351.

[16] A. H. Dhalla, D. Nankivil, T. Bustamante, A. Kuo, J. A. Izatt, Opt. Lett. 2012, 37(11), 1883.

[17] W. J. Choi, R. K. Wang, Quantum Electron. 2014, 44(8), 740.

[18] W. J. Choi, R. K. Wang, Biomed. Opt. Express 2014, 5(8), 2620.

[19] M. Paukert, D. E. Bergles, J. Physiol. 2012, 590(13), 2955.
[20] S. Yousefi, J. Qin, Z. Zhi, R. K. Wang, Quant. Imaging Med. Surg. 2013, 3(1), 5 .

[21] R. Reif, S. Yousefi, W. J. Choi, R. K. Wang, Appl. Opt. 2014, 53 (5), 806

[22] Y. Jia, O. Tan, J. Tokayer, B. Potsaid, Y. Wang, J. J. Liu, M. F. Kraus, H. Subhash, J. G. Fujimoto, J. Hornegger, D. Huang, Opt. Express 2012, 20(4), 4710.

[23] H. Hendargo, R. Estrada, S. Chiu, C. Tomasi, S. Farsiu, J. Izatt, Invest. Ophthalmol. Vis. Sci. 2013, 54(15), 5528.

[24] R. Reif, U. Baran, R. K. Wang, Appl. Opt. 2014, 53(19), 4164.

[25] Y. Watanabe, Y. Takahashi, H. Numazawa, J. Biomed. Opt. 2013, 19(2), 021105.

[26] G. Liu, L. Chou, W. Jia, W. Qi, B. Choi, Z. Chen, Opt. Express 2011, 19(12), 11429

[27] T. Vercauteren, X. Pennec, A. Perchant, N. Ayache, NeuroImage 2009, 45(1), S61.

[28] T. Vercauteren, X. Pennec, A. Perchant, N. Ayache, International Conference on Medical Image Computing and Computer-Assisted Intervention, Springer, Berlin 2008, p. 754.

[29] J. Kittler, J. Illingworth, Pattern Recogn. 1986, 19(1), 41.

[30] Z. Ma, N. Ding, Y. Yu, Y. Ma, X. Yuan, Y. Wang, J. Liu, Appl. Opt. 2018, 57(35), 10117.

[31] J. Liu, Y. Ma, S. Dou, Y. Wang, D. La, J. Liu, Z. Ma, J. Biomed. Opt. 2016, 21(7), 075014.

[32] J. Liu, N. Ding, Y. Yu, X. Yuan, S. Luo, J. Luan, Z. Ma, J. Biomed. Optics 2019, 24(3), 035002.

[33] P. K. Manwaring, K. L. Moodie, A. Hartov, K. H. Manwaring, R. J. Halter, Anesth. Analg. 2013, 117(4), 866.

[34] H. L. Lagreze, R. L. Levine, K. L. Pedula, R. J. Nickles, J. S. Sunderland, B. R. Rowe, Stroke 1987, 18(5), 882.

[35] G. Rubin, E. I. Levy, A. M. Scarrow, A. D. Firlik, A. din Karakus, L. R. Wechsler, H. Yonas, Cerebrovasc. Dis. 2000, 10(3), 221.

[36] H. K. Kimelberg, Neuroscience 2000, 6, 14.

[37] J. M. Simard, T. A. Kent, M. Chen, K. V. Tarasov, V. Gerzanich, Lancet Neurol. 2007, 6(3), 258.

[38] A. W. Vorbrodt, A. S. Lossinsky, H. M. Wisniewski, R. Suzuki, T. Yamaguchi, H. Masaoka, I. Klatzo, Acta Neuropathol. 1985, 66(4), 265

[39] J. J. Donkin, R. Vink, Curr. Opin. Neurol. 2010, 23(3), 293.

How to cite this article: Liu J, Ding N, Yu Y, et al. Whole-brain microcirculation detection after ischemic stroke based on swept-source optical coherence tomography. J. Biophotonics. 2019;12:e201900122. https://doi.org/10.1002/jbio.201900122 